\title{
Tire Radii Estimation Using a Marginalized Particle Filter
}

\author{
Christian Lundquist, Rickard Karlsson, Emre Özkan and Fredrik Gustafsson
}

\section{Linköping University Post Print}

\section{Tweet}

N.B.: When citing this work, cite the original article.

(C2014 IEEE. Personal use of this material is permitted. However, permission to reprint/republish this material for advertising or promotional purposes or for creating new collective works for resale or redistribution to servers or lists, or to reuse any copyrighted component of this work in other works must be obtained from the IEEE.

Christian Lundquist, Rickard Karlsson, Emre Özkan and Fredrik Gustafsson, Tire Radii Estimation Using a Marginalized Particle Filter, 2014, IEEE transactions on intelligent transportation systems (Print), (15), 2, 663-672.

http://dx.doi.org/10.1109/TITS.2013.2284930

Postprint available at: Linköping University Electronic Press http://urn.kb.se/resolve?urn=urn:nbn:se:liu:diva-106514 


\title{
Tire Radii Estimation Using a Marginalized Particle Filter
}

\author{
Christian Lundquist, Rickard Karlsson, Emre Özkan, Member, IEEE, and Fredrik Gustafsson, Fellow, IEEE,
}

\begin{abstract}
In this work measurements of individual wheel speeds and absolute position from a global positioning system (GPS) are used for high-precision estimation of vehicle tire radii. The radii deviation from its nominal value is modeled as a Gaussian random variable and included as noise components in a simple vehicle motion model. The novelty lies in a Bayesian approach to estimate online both the state vector and the parameters representing the process noise statistics using a marginalized particle filter. Field tests show that the absolute radius can be estimated with sub-millimeter accuracy. The approach is tested in accordance with the ECE $\mathrm{R}-64$ regulation on a large data set (22 tests, using two vehicles and 12 different tire sets), where tire deflations are detected successfully, with high robustness, i.e., no false alarms. The proposed marginalized particle filter approach outperforms common Kalman filter based methods used for joint state and parameter estimation when compared with respect to accuracy and robustness.
\end{abstract}

Index Terms-marginalized particle filter, tire radius, conjugate prior, noise parameter estimation.

\section{INTRODUCTION}

$\mathbf{T}$ IRE pressure monitoring has become an integral part of todays' automotive active safety concept [1]. With the announcement of the US standard (FMVSS 138) and the European standard (ECE R-64), vehicle manufacturer must provide a robust solution to early detect tire pressure loss. A direct way to measure the tire pressure is to equip the wheel with a pressure sensor and transmit the information wireless to the vehicle body. In the harsh environment that the tires are exposed to, e.g., water, salt, ice and heat, the sensors are errorprone. Furthermore, the direct system with pressure sensors in each wheel, wirelessly communicating with the body, is expensive and the existence of sensors complicates the wheel changes. Therefore, indirect solutions have been introduced on the market lately, and are today implemented in more than 5 million passenger cars. In this paper, an indirect approach is presented where the tire radius is estimated simultaneously with the vehicle trajectory. The estimation is based on the relation between a reduction in tire radius and a reduction tire pressure.

The indirect approach presented in [2] is only based on the wheel speed sensors and it is shown how a tire pressure

The authors gratefully acknowledge the fundings from the Swedish Research Council under the Linnaeus Center CADICS and under the frame project grant Extended Target Tracking (621-2010-4301).

Christian Lundquist, Emre Özkan and Fredrik Gustafsson are with the Department of Electrical Engineering, Division of Automatic Control, Linköping University, SE-581 83 Linköping, Sweden, (e-mail: lundquist@isy.liu.se; emre@isy.liu.se; fredrik@isy.liu.se).

Rickard Karlsson is with Nira Dynamics AB, SE-583 30 Linköping, Sweden, (e-mail: rickard.karlsson@ niradynamics.se). loss in one wheel leads to a relative radii error between the wheels. An advantage is that the wheel speed sensors are an important part of standard ABS (anti-lock braking system) and that all cars are therefore already equipped with these sensors. In later publications GPS measurements have also been included to improve the radius estimation, which even make estimating of the absolute radius of one tire possible. The effective tire radius is estimated using a simple least-squares regression technique in [3]. A nonlinear observer approach to estimate the tire radius is presented in [4], and a second order sliding mode observer is used to estimate the tire radius in [5] and [6]. A simultaneous maximum likelihood calibration and sensor position and orientation estimation approach for mobile robots is presented in [7], where among other parameters the wheel radii are estimated. An observer based fault detection algorithm, which detects tire radius errors using yaw rate measurement and a bicycle model of the vehicle, is described in [8]. An extended Kalman filter based approach is presented in [9], where the tire radius is estimated via vertical chassis accelerations.

In the present contribution the difference between the static radius and the rolling radius of the tire is modeled as a Gaussian random variable, where both the mean and the covariance are unknown and time varying. Each wheel is treated separately, not relative to each other, hence it is possible to detect tire pressure loss in all four wheels using the proposed method. The vehicle dynamics and the measurements are described by a general state space model and the noise statistics are treated as the unknown parameters of the model. Hence a joint estimation of the state vector and the unknown model parameters based on available measurements is required. Such a problem is hard to treat as both the state estimation and the parameter estimation stages effects the performance of the other. The model used in this paper is nonlinear with biased and unknown noise, which requires approximate estimation algorithms. The particle filter ( $\mathrm{PF}),[10]-[12]$, provides one generic approach to nonlinear non-Gaussian filtering. Probably the most common way to handle a joint parameter and state estimation problem is by augmenting the state vector with the unknown parameters and redefine the problem as a filtering problem, see e.g., [13]-[15]. A state augmentation approach has some major disadvantages as it requires artificial dynamics for the static parameters and it leads also to an increase in the state dimension which is not preferable for particle filters. This is particularly important to stress in automotive applications, where the computational cost must be kept low. In this work, an efficient Bayesian method is proposed for approximating the joint density of the unknown parameters and the state 
based on the particle filters and the marginalization concept, introduced in [16]. The statistics of the posterior distribution of the unknown noise parameters are propagated recursively conditional on the nonlinear states of the particle filter. An earlier version of this work was presented in [17], and the current, significantly extended version, includes comparisons with other methods, a more comprehensive evaluation based on a large number of data sets and an algorithm to detect tire deflation.

The proposed method is implemented and tested on real vehicle data utilizing several different tires. The state augmentation technique is also implemented and tested, using both the $\mathrm{PF}$ and the extended Kalman filter (EKF) variants.

The paper is outlined as follows. The problem is formulated together with the vehicle model and with the description of the sensors in Section II. The marginalized particle filter approach is described in Section III. Results based on real data collected with a production type passenger car are presented in Section IV. The work is concluded in Section V.

\section{MODEL}

The aim of this work is to jointly estimate the vehicle trajectory and the unknown tire radii errors based on the available measurements in a Bayesian framework. The proposed estimation approach is model based and a general state space model is given by

$$
\begin{aligned}
\mathbf{x}_{k+1} & =f\left(\mathbf{x}_{k}, \mathbf{u}_{k}\right)+g\left(\mathbf{x}_{k}, \mathbf{u}_{k}\right) \mathbf{w}_{k}, \\
\mathbf{y}_{k} & =h\left(\mathbf{x}_{k}\right)+\mathbf{e}_{k} .
\end{aligned}
$$

where $\mathbf{x}$ is the state vector, $\mathbf{u}$ is the deterministic measurement signal, $\mathbf{y}$ is the stochastic measurements signal, $\mathbf{w}$ is the process noise and $\mathbf{e}$ is the measurement noise. Further, $k$ is the discrete time counter.

It is assumed that the unknown wheel radii affect the expected vehicle state trajectory through the wheel speed sensors, since a wheel with reduced radius must turn faster to travel the same distance. Hence, measurement of wheel revolutions from the wheel speed sensors and the trajectory from the GPS are used in the proposed approach. The angular velocities $\omega$ of the wheels are considered as deterministic measurement signals $\mathbf{u}$ under the assumption that the measurement noise of these signals are negligible. The GPS positions are considered as measurement signals $\mathbf{y}$.

The state vector is defined as

$$
\mathbf{x}=\left[\begin{array}{lll}
x & y & \psi
\end{array}\right]^{\mathrm{T}},
$$

where $\mathrm{x}, \mathrm{y}$ is the planar position and $\psi$ is the heading angle of the vehicle. The tire radii deviations from the nominal values are considered as time varying parameters and later in this section it is shown how the parameters are integrated in the state space model (1).

To begin with, the discrete time motion model (1a) is derived. The tire radii are primarily estimated during normal and non-extreme driving on paved roads, hence a very simple motion model is sufficient to describe the vehicle motion, which is also beneficial from a computational point of view. The model is given by

$$
\begin{aligned}
\mathrm{x}_{k+1} & =\mathrm{x}_{k}+T \mathrm{v}_{k} \cos \psi_{k} \\
\mathrm{y}_{k+1} & =\mathrm{y}_{k}+T \mathrm{v}_{k} \sin \psi_{k} \\
\psi_{k+1} & =\psi_{k}+T \dot{\psi}_{k},
\end{aligned}
$$

where $v_{k}$ is the vehicle longitudinal velocity, $\dot{\psi}_{k}$ is the yaw rate of the vehicle and $T$ is the sampling time. A more advanced vehicle model would be the bicycle model, also denoted single track model, see e.g., [18], [19]. It describes the vehicle motion better and more accurate but it has the disadvantages that it both requires more parameters, such as cornering stiffness which must be identified online, and it has a higher state dimension.

For a wheel, there exist primarily three different definitions of the wheel radius, see e.g., [19]. The static tire radius is the distance between the center of the wheel and the ground. The rolling radius is the radius of a free rolling tire and it is defined as the linear speed of the tire center divided by the angular speed of the tire. Finally, the effective rolling radius is the radius of a driven wheel, which is smaller than the rolling radius of a free rolling wheel because of slip. In this work a front wheel driven vehicle is assumed and only the angular velocity measurements from the free rolling rear wheels are used to avoid slip estimation. The angular velocities of the rear wheels $\omega_{i}, i=3,4$ can be converted to virtual measurements of the absolute longitudinal velocity and yaw rate as described in [20], [21], according to

$$
\begin{aligned}
\mathrm{v}^{\mathrm{virt}} & =\frac{\omega_{3} r+\omega_{4} r}{2} \\
\dot{\psi}^{\mathrm{virt}} & =\frac{\omega_{4} r-\omega_{3} r}{l_{T}},
\end{aligned}
$$

where $r$ is the static wheel radius and $l_{T}$ is the wheel track; see Fig. 1 for the notation. The static radius, given by the tire manufacturer, differs from the rolling radius, which in reality is unknown and needs to be estimated on the run. The wheel radius errors, are the important parameters to be estimated, and they are defined as the difference between the rolling radius and the static radius of the rear left and right wheels $\delta_{3} \triangleq r_{3}-r$ and $\delta_{4} \triangleq r_{4}-r$, respectively. In this paper, we have simplified the model, studying only the rear axle. The model can be extended with the front axle radii if a complete slip model is introduced. The rolling tire radii are

$$
\begin{aligned}
& r_{3}=r+\delta_{3} \\
& r_{4}=r+\delta_{4} .
\end{aligned}
$$

Multiplying $r_{3}$ with $\omega_{3}$ and $r_{4}$ with $\omega_{4}$ in equations (4) to derive the longitudinal velocity $\mathrm{v}$ and yaw rate $\psi$ results in

$$
\begin{aligned}
& \mathrm{v}=\frac{\omega_{3} r_{3}+\omega_{4} r_{4}}{2}=\mathrm{v}^{\mathrm{virt}}+\frac{\omega_{3} \delta_{3}}{2}+\frac{\omega_{4} \delta_{4}}{2} \\
& \dot{\psi}=\frac{\omega_{4} r_{4}-\omega_{3} r_{3}}{l_{T}}=\dot{\psi}^{\mathrm{virt}}+\frac{\omega_{4} \delta_{4}}{l_{T}}-\frac{\omega_{3} \delta_{3}}{l_{T}} .
\end{aligned}
$$




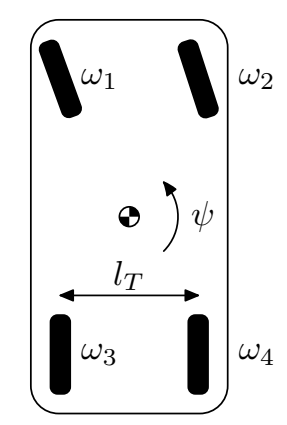

Fig. 1. The front wheels are denoted 1 and 2, and the rear wheels of the vehicle are denoted 3 and 4 . Further the yaw angle is defined around the center of gravity, denoted $\otimes$.

Substituting these into the motion model (3) gives

$$
\begin{aligned}
& \mathrm{x}_{k+1}=\mathrm{x}_{k}+T\left(\mathrm{v}_{k}^{\mathrm{virt}}+\frac{\omega_{3, k} \delta_{3, k}}{2}+\frac{\omega_{4, k} \delta_{4, k}}{2}\right) \cos \psi_{k} \\
& \mathrm{y}_{k+1}=\mathrm{y}_{k}+T\left(\mathrm{v}_{k}^{\text {virt }}+\frac{\omega_{3, k} \delta_{3, k}}{2}+\frac{\omega_{4, k} \delta_{4, k}}{2}\right) \sin \psi_{k} \\
& \psi_{k+1}=\psi_{k}+T\left(\dot{\psi}_{k}^{\text {virt }}+\frac{\omega_{4, k} \delta_{4, k}}{l_{T}}-\frac{\omega_{3, k} \delta_{3, k}}{l_{T}}\right),
\end{aligned}
$$

where the input vector $\mathbf{u}$ only consists of the wheel speeds, i.e.,

$$
\mathbf{u}_{k}=\left[\begin{array}{ll}
\omega_{3, k} & \omega_{4, k}
\end{array}\right]^{\mathrm{T}}
$$

since $\mathrm{v}_{k}^{\text {virt }}$ and $\dot{\psi}_{k}^{\text {virt }}$ are functions of the wheel speeds.

At this point, the observability of the parameters should be analyzed. Consider a simple example, where the numbers are taken from our test vehicle shown in Fig. 2. The rear left tire radius is reduced by $1 \mathrm{~mm}$, i.e., $\delta_{3}=-0.001$, and without loss of generality the radius of the rear right wheel is kept without radius reduction, i.e., $\delta_{4}=0$. The nominal tire radius is $0.27 \mathrm{~m}$, the wheel track is $l_{T}=1.52 \mathrm{~m}$ and the wheel revolutions are $\omega_{3}=76 \mathrm{rad} / \mathrm{s}$ and $\omega_{4}=75.8 \mathrm{rad} / \mathrm{s}$. The vehicle velocity according to (6a) is then

$$
\vee=0.27 \frac{76+75.8}{2}-0.001 \frac{76}{2}=\underbrace{20.5}_{\text {virtual }}-\underbrace{0.038}_{\text {error }},
$$

i.e., the wheel radii error is approximately $0.2 \%$ of the virtual velocity. Now consider the yaw rate according to (6b)

$$
\dot{\psi}=0.27 \frac{75.8-76}{1.52}+0.001 \frac{76}{1.52}=\underbrace{-0.036}_{\text {virtual }}+\underbrace{0.05}_{\text {error }}
$$

where the wheel radii error is approximately $140 \%$ of the virtual yaw rate. Note that the sum of errors is considered in (9a) and that the difference is considered in (9b). This example shows that it is much easier to estimate the tire radii difference between the left and the right wheel than to estimate the sum and thereby the absolute value of them.

The time varying parameters $\delta_{3}$ and $\delta_{4}$ are integrated in the motion model (7). One common approach in joint state and parameter estimation is to augment the state vector with the unknown parameters [15]. In such case, the augmented state vector is defined as

$$
\mathbf{x}^{a}=\left[\begin{array}{lllll}
\mathbf{x} & \mathrm{y} & \psi & \delta_{3} & \delta_{4}
\end{array}\right]^{\mathrm{T}},
$$

and the state space model can be written in the general form (1) with the components according to

$$
\begin{aligned}
& f\left(\mathbf{x}_{k}^{a}, \mathbf{u}_{k}\right)= {\left[\begin{array}{c}
\mathrm{x}_{k}+T\left(\mathrm{v}_{k}^{\mathrm{virt}}+\frac{\omega_{3, k} \delta_{3, k}+\omega_{4, k} \delta_{4, k}}{2}\right) \\
\mathrm{y}_{k}+T\left(\mathrm{v}_{k}^{\mathrm{virt}}+\frac{\omega_{3, k} \delta_{3, k}+\omega_{4, k} \delta_{4, k}}{2}\right) \cos \psi_{k} \\
\psi_{k}+T\left(\dot{\psi}_{k}^{\mathrm{virt}}-\frac{\omega_{3, k} \delta_{3, k}-\omega_{4, k} \delta_{4, k}}{l_{T}}\right) \\
\delta_{3, k} \\
\delta_{4, k}
\end{array}\right] } \\
& g\left(\mathbf{x}_{k}^{a}, \mathbf{u}_{k}\right)=\left[\begin{array}{cccc}
\frac{T \omega_{3, k} \cos \psi_{k}}{2} & \frac{T \omega_{4, k} \cos \psi_{k}}{2} & 0 & 0 \\
\frac{T \omega_{3, k} \sin \psi_{k}}{2} & \frac{T \omega_{4, k} \sin \psi_{k}}{2} & 0 & 0 \\
-\frac{T \omega_{3, k}}{l_{T}} & \frac{T \omega_{4, k}}{l_{T}} & 0 & 0 \\
0 & 0 & 1 & 0 \\
0 & 0 & 0 & 1
\end{array}\right]
\end{aligned}
$$

The process noise $\mathbf{w}_{k}$ is zero mean Gaussian noise. i.e.,

$$
\mathbf{w}=\mathcal{N}(0, Q) .
$$

and it will influence the parameters $\delta_{3}$ and $\delta_{4}$ directly and the position and orientation of the vehicle through the noise model (11b), as if the noise was associated with the parameters. The presented rather simple vehicle model is accurate enough for tire radii estimation when the vehicle is not maneuvering or accelerating too much. Hence, in order to keep the model simple, estimation disable criteria, for instance dynamic driving, braking and velocity constraints are introduced.

The augmented state vector (10) can be estimated using e.g., an EKF. The observability can also be analyzed by deriving the observability Gramian, [22]. Since the model (11) is nonlinear and discrete it must be linearized to derive the Gramian. The Gramian is non-singular for the present system, hence weakly observable.

Another solution would be to use a particle filter (PF) instead of an EKF to estimate the states. An advantage with the $\mathrm{PF}$ is that the state space easily can be constrained, to not allow obvious estimation errors, such as a non negative radius error caused by tire deflation. However, the augmented state vector with 5 states is too large to obtain good results without the need to use a large number of particles. A marginalized approach has recently been published in [16], which makes it possible to keep a state vector of size 3, obtaining good results with a low number of particles and keeping the computational cost low. The marginalized PF approach is summarized in Section III. Using that approach, the motion model (7) can be written in the form (1a), where the functions $f$ and $g$ are identified as

$$
\begin{aligned}
f\left(\mathbf{x}_{k}, \mathbf{u}_{k}\right) & =\left[\begin{array}{c}
\mathrm{x}_{k}+T \mathrm{v}_{k}^{\text {virt }} \cos \psi_{k} \\
\mathrm{y}_{k}+T \mathbf{v}_{k}^{\text {virt }} \sin \psi_{k} \\
\psi_{k}+T \dot{\psi}_{k}^{\text {virt }}
\end{array}\right] \\
g\left(\mathbf{x}_{k}, \mathbf{u}_{k}\right) & =\left[\begin{array}{cc}
\frac{T \omega_{3, k} \cos \psi_{k}}{2} & \frac{T \omega_{4, k} \cos \psi_{k}}{2} \\
\frac{T \omega_{3, k} \sin \psi_{k}}{2} & \frac{T \omega_{4, k} \sin \psi_{k}}{2} \\
-\frac{T \omega_{3, k}}{l_{T}} & \frac{T \omega_{4, k}}{l_{T}}
\end{array}\right]
\end{aligned}
$$

and where the process noise term $\mathbf{w}_{k}$ contains the time varying 
parameters and is given as,

$$
\mathbf{w}_{k}=\left[\begin{array}{l}
\delta_{3, k} \\
\delta_{4, k}
\end{array}\right] \sim \mathcal{N}\left(\mu_{k}, \Sigma_{k}\right)=\mathcal{N}\left(\left[\begin{array}{l}
\mu_{3, k} \\
\mu_{4, k}
\end{array}\right],\left[\begin{array}{cc}
\sigma_{3, k}^{2} & 0 \\
0 & \sigma_{4, k}^{2}
\end{array}\right]\right) .
$$

The process noise, or the tire radius error, is described by two parameters, the mean value $\mu$ and the covariance $\Sigma$ for the left and the right wheel. Intuitively the mean value corresponds to the slow time variations from the nominal tire radius, while the variance corresponds to the fast variations due to tire vibrations. One interpretation is that the mean value $\mu$ models the change in the wheel radii due to abrasion, tire pressure changes and effects of cornering, and the covariance $\Sigma$ can account for the vibrations arising from an uneven road surface. An interesting special case is when $\sigma_{3}=\sigma_{4}$, which represents homogeneous road conditions, in comparison with split road surface when $\sigma_{3} \neq \sigma_{4}$.

The measurement model (1b) defines the relation between the GPS position measurements and the state variables as follows.

$$
\begin{aligned}
\mathbf{y}_{k} & =\left[\begin{array}{ll}
\mathrm{x}_{k}^{\mathrm{GPS}} & \mathrm{y}_{k}^{\mathrm{GPS}}
\end{array}\right]^{\mathrm{T}} \\
h\left(\mathbf{x}_{k}\right) & =\left[\begin{array}{ll}
I & 0_{2 \times\left(n_{\mathbf{x}}-2\right)}
\end{array}\right] \mathbf{x}_{k}
\end{aligned}
$$

where $0_{2 \times\left(n_{\mathbf{x}}-2\right)}$ is a zero matrix, where $n_{\mathbf{x}}=3$ for the regular state space model and $n_{\mathbf{x}}=5$ for the augmented state space model. The measurement noise is assumed to be Gaussian with zero mean and known constant covariance $R$, i.e.,

$$
\mathbf{e}_{k}=\left[\begin{array}{ll}
\mathbf{e}_{\mathrm{x}} & \mathbf{e}_{\mathrm{y}}
\end{array}\right]^{\mathrm{T}}=\mathcal{N}(0, R), \quad R=\sigma_{\mathrm{GPS}}^{2} I_{2}
$$

where $\sigma_{\mathrm{GPS}}$ is the standard deviation of the GPS measurements. Other sensor measurements are also plausible to include in the measurement vector, at the cost of a more complex model. For instance, using a yaw rate gyro to measure the third state, also requires modeling the drifting offset in the sensor. The steering angle can also be converted to yaw rate, but it suffers from dynamic lag and other dynamic states of the vehicle.

\section{MARginalized PARTICLE Filter For PARAMETER ESTIMATION}

This section focuses on the evaluation of the joint density $p\left(\mathbf{x}_{k}, \boldsymbol{\theta}_{k} \mid \mathbf{y}_{1: k}\right)$ of the state variable $\mathbf{x}_{k}$ defined in (2) and the parameters $\boldsymbol{\theta}_{k}$, that are the radii error biases and the covariances according to,

$$
\boldsymbol{\theta}_{k} \triangleq\left\{\mu_{3, k}, \mu_{4, k}, \sigma_{3, k}, \sigma_{4, k}\right\},
$$

conditioned on all measurements $\mathbf{y}_{1: k}$ from time 1 to $k$. In order to simplify the calculations, the target density is decomposed into conditional densities as follows

$$
p\left(\mathbf{x}_{0: k}, \boldsymbol{\theta}_{k} \mid \mathbf{y}_{1: k}\right)=p\left(\boldsymbol{\theta}_{k} \mid \mathbf{x}_{0: k}, \mathbf{y}_{1: k}\right) p\left(\mathbf{x}_{0: k} \mid \mathbf{y}_{1: k}\right) .
$$

The resulting two densities are estimated recursively. The implementation of the estimation algorithm is described in three steps. In Section III-A we define the approximations made to derive the state trajectory $p\left(\mathbf{x}_{0: k} \mid \mathbf{y}_{1: k}\right)$ and in Section III-B we describe the estimation of the sufficient statistics of the parameter distribution $p\left(\boldsymbol{\theta}_{k} \mid \mathbf{x}_{0: k}, \mathbf{y}_{1: k}\right)$. The predicted trajectory is derived conditioned on the parameter estimates and the estimation of the joint density and the marginal density of the states $p\left(\mathbf{x}_{0: k} \mid \mathbf{y}_{1: k}\right)$ is finalized in Section III-C.

\section{A. State Trajectory}

The posterior distribution of state trajectory $p\left(\mathbf{x}_{0: k} \mid \mathbf{y}_{1: k}\right)$ is approximated by an empirical density of $N$ particles and their weights as follows:

$$
p\left(\mathbf{x}_{0: k} \mid \mathbf{y}_{1: k}\right) \approx \sum_{i=1}^{N} w_{k}^{(i)} \delta\left(\mathbf{x}_{0: k}-\mathbf{x}_{0: k}^{(i)}\right)
$$

where $\mathbf{x}_{0: k}^{(i)}$ is a trajectory sample and $w_{k}^{(i)}$ is its related weight. The approximate state trajectory distribution (16) is propagated with a particle filter, using the sequential importance sampling (SIS) scheme [23]. In this scheme, at any time $k$, first the samples, which are also denoted by particles, are generated from a proposal distribution $q\left(\mathbf{x}_{k} \mid \mathbf{x}_{0: k-1}^{(i)}, \mathbf{y}_{1: k}\right)$ by using the particles from time $k-1$. The sampling step is followed by the update step in which the weights are updated according to

$$
w_{k}^{(i)} \propto \frac{p\left(\mathbf{y}_{k} \mid \mathbf{x}_{k}^{(i)}\right) p\left(\mathbf{x}_{k} \mid \mathbf{x}_{0: k-1}^{(i)}, \mathbf{y}_{1: k-1}\right)}{q\left(\mathbf{x}_{k} \mid \mathbf{x}_{0: k-1}^{(i)}, \mathbf{y}_{1: k}\right)} w_{k-1}^{(i)} .
$$

where $p\left(\mathbf{y}_{k} \mid \mathbf{x}_{k}^{(i)}\right)$ is the likelihood function. Furthermore, if the proposal distribution $q\left(\mathbf{x}_{k} \mid \mathbf{x}_{0: k-1}^{(i)}, \mathbf{y}_{1: k}\right)$ is chosen as the prediction distribution $p\left(\mathbf{x}_{k} \mid \mathbf{x}_{0: k-1}^{(i)}, \mathbf{y}_{1: k-1}\right)$, then the weight update reduces to,

$$
w_{k}^{(i)}=w_{k-1}^{(i)} p\left(\mathbf{y}_{k} \mid \mathbf{x}_{k}^{(i)}\right) .
$$

The prediction and update steps of the parameter estimation are described in the next section and the joint recursive estimation of both states and estimation are stringed together in Section III-C.

\section{B. Parameter Estimation}

In the factorization of the joint density given in equation (15), the distribution of the unknown parameters (which corresponds to the first term) is computed conditional on the realization of the state trajectory and the measurements. For a given sample $\mathbf{x}_{0: k}^{(i)}$ the motion model (1a) may be rewritten according to

$$
\mathbf{w}_{k}^{(i)}=g^{-\dagger}\left(\mathbf{x}_{k-1}^{(i)}, \mathbf{u}_{k-1}\right)\left(\mathbf{x}_{k}^{(i)}-f\left(\mathbf{x}_{k-1}^{(i)}, \mathbf{u}_{k-1}\right)\right),
$$

where $\dagger$ is the pseudo inverse. Hence, for a specific realization $\mathbf{x}_{0: k}^{(i)}$ of the trajectory, the posterior density of the parameters can also be written conditional on the realization of the noise terms according to

$$
p\left(\boldsymbol{\theta}_{k} \mid \mathbf{x}_{0: k}^{(i)}, \mathbf{y}_{1: k}\right)=p\left(\boldsymbol{\theta}_{k} \mid \mathbf{w}_{0: k}^{(i)}\right) .
$$

Furthermore, this posterior can be decomposed into a likelihood function and a prior according to Bayes rule

$$
p\left(\boldsymbol{\theta}_{k} \mid \mathbf{w}_{0: k}\right) \propto p\left(\mathbf{w}_{k} \mid \boldsymbol{\theta}_{k}\right) p\left(\boldsymbol{\theta}_{k} \mid \mathbf{w}_{0: k-1}\right) .
$$

The likelihood function $p\left(\mathbf{w}_{k} \mid \boldsymbol{\theta}_{k}\right)$ is assumed to be multivariate Gaussian, as previously mentioned in (12c), and since 
the mean $\mu_{k}$ and the covariance $\Sigma_{k}$ are considered unknown parameters $\boldsymbol{\theta}_{k}$, a Normal-inverse-Wishart distribution defines the conjugate prior $^{1} p\left(\boldsymbol{\theta}_{k} \mid \mathbf{w}_{0: k-1}\right)$. Normal-inverse-Wishart distribution defines a hierarchical Bayesian model given as

$$
\begin{aligned}
\mathbf{w}_{k} \mid \mu_{k}, \Sigma_{k} & \sim \mathcal{N}\left(\mu_{k}, \Sigma_{k}\right) \\
\mu_{k} \mid \Sigma_{k} & \sim \mathcal{N}\left(\hat{\mu}_{k \mid k}, \gamma_{k \mid k} \Sigma_{k \mid k}\right) \\
\Sigma_{k} & \sim \mathrm{iW}\left(\nu_{k \mid k}, \Lambda_{k \mid k}\right) \\
& \propto\left|\Sigma_{k}\right|^{-\frac{1}{2}(\nu+d+1)} \exp \left(-\frac{1}{2} \operatorname{Tr}\left(\Lambda_{k \mid k} \Sigma_{k}^{-1}\right)\right)
\end{aligned}
$$

where $\mathrm{iW}($.$) denotes the inverse Wishart distribution and d$ denotes the dimension of the noise vector $\mathbf{w}_{k}$. The statistics $S_{\mathbf{w}, k} \triangleq\left\{\gamma_{k}, \hat{\mu}_{k}, \nu_{k}, \Lambda_{k}\right\}$ can according to [24], [25] be recursively updated as follows. The measurement update is

$$
\begin{aligned}
\gamma_{k \mid k} & =\frac{\gamma_{k \mid k-1}}{1+\gamma_{k \mid k-1}} \\
\hat{\mu}_{k \mid k} & =\hat{\mu}_{k \mid k-1}+\gamma_{k \mid k}\left(\mathbf{w}_{k}-\hat{\mu}_{k \mid k-1}\right) \\
\nu_{k \mid k} & =\nu_{k \mid k-1}+1 \\
\Lambda_{k \mid k} & =\Lambda_{k \mid k-1}+\frac{1}{1+\gamma_{k \mid k-1}}\left(\hat{\mu}_{k \mid k-1}-\mathbf{w}_{k}\right)\left(\hat{\mu}_{k \mid k-1}-\mathbf{w}_{k}\right)^{\mathrm{T}}
\end{aligned}
$$

where the statistics of the predictive distributions are given by the time update step according to

$$
\begin{aligned}
\gamma_{k \mid k-1} & =\frac{1}{\lambda} \gamma_{k-1 \mid k-1} \\
\hat{\mu}_{k \mid k-1} & =\hat{\mu}_{k-1 \mid k-1} \\
\nu_{k \mid k-1} & =\lambda \nu_{k-1 \mid k-1} \\
\Lambda_{k \mid k-1} & =\lambda \Lambda_{k-1 \mid k-1} .
\end{aligned}
$$

The scalar real number $0 \leq \lambda \leq 1$ is the forgetting factor. The forgetting factor here helps in the estimation of the dynamic variables. The statistics relies on roughly the measurements within the last $h=\frac{1}{1-\lambda}$ frames/time instances. That allows the algorithm to adapt the changes in the noise statistics in time. Such an approach is appropriate when the unknown parameters are slowly varying, and the underlying parameter evolution is unknown.

\section{Noise Marginalization}

In this section the two densities in (15) will be unified into one iterative estimation scheme. Consider first the state prediction and note that by using (19) it may be rewritten using the lemma on transformation of variables in probability density functions according to

$$
p\left(\mathbf{x}_{k} \mid \mathbf{x}_{0: k-1}^{(i)}, \mathbf{y}_{1: k-1}\right) \propto p\left(\mathbf{w}\left(\mathbf{x}_{k}\right) \mid S_{\mathbf{w}, k}^{(i)}\right)
$$

Using the marginalization concept it may also be rewritten as

$$
\begin{aligned}
& p\left(\mathbf{x}_{k} \mid \mathbf{x}_{0: k-1}^{(i)}, \mathbf{y}_{1: k-1}\right) \\
& \quad=\int p\left(\mathbf{x}_{k} \mid \mathbf{x}_{k-1}^{(i)}, \boldsymbol{\theta}\right) p\left(\boldsymbol{\theta} \mid \mathbf{x}_{0: k-1}^{(i)}, \mathbf{y}_{1: k-1}\right) d \boldsymbol{\theta} .
\end{aligned}
$$

\footnotetext{
${ }^{1}$ A family of prior distributions is conjugate to a particular likelihood function if the posterior distribution belongs to the same family as the prior.
}

One important advantage of using the conjugate priors reveals itself here as it is possible to integrate out the unknown noise parameters as they follow Normal-inverse-Wishart distribution. The integrand in (26) is the product of a Gaussian distribution and a NiW distribution and the result of the integral is a Student-t distribution which can be evaluated analytically.

Now, by combining (25) and (26), the predictive distribution of $\mathbf{w}_{k}$ becomes a multivariate Student-t density, according to

$$
p\left(\mathbf{w}_{k} \mid S_{\mathbf{w}, k}^{(i)}\right)=\operatorname{St}\left(\hat{\mu}_{k \mid k-1}^{(i)}, \Lambda_{k \mid k-1}^{(i)}, \nu_{k \mid k-1}^{(i)}-d+1\right)
$$

with $\nu_{k \mid k-1}^{(i)}-d+1$ degrees of freedom. The Student-t distribution is located at $\hat{\mu}_{k \mid k-1}^{(i)}$ with variance

$$
\operatorname{Var}\left(\mathbf{w}_{k}\right)=\frac{1+\gamma_{k \mid k-1}}{\nu_{k \mid k-1}-d-1} \Lambda_{k \mid k-1}
$$

where the relevant statistics are given in (24).

In the implementation, the noise is first sampled from (27) and used in (1a) in order to create samples $\mathbf{x}_{k}^{(i)}$. The samples from (27) can be used directly in the statistics update (23). The pseudo code of the algorithm used in the simulations is given in Table I.

In the proposed method, each particle $i$ keeps its own estimate for the parameters $\boldsymbol{\theta}^{(i)}$ of the unknown process noise. In the importance sampling step, the particles use their own posterior distribution of the unknown parameters. The weight update of the particles is performed according to the measurement likelihood. The particles are keeping the unknown parameters, and those which best explain the observed measurement sequence will survive in time.

The marginal posterior density of the unknown parameters can be computed by integrating out the states in the joint density

$$
\begin{aligned}
p\left(\boldsymbol{\theta} \mid \mathbf{y}_{1: k}\right) & =\int p\left(\boldsymbol{\theta} \mid \mathbf{x}_{0: k}, \mathbf{y}_{1: k}\right) p\left(\mathbf{x}_{0: k} \mid \mathbf{y}_{1: k}\right) d \mathbf{x}_{0: k} \\
& \approx \sum_{i=1}^{N} \omega_{k}^{(i)} p\left(\boldsymbol{\theta} \mid \mathbf{x}_{0: k}^{(i)}, \mathbf{y}_{1: k}\right) .
\end{aligned}
$$

Then the estimate of the unknown parameters could be computed according to a chosen criterion. As an example, according to the minimum mean square error (MMSE) criterion, the noise mean and variance estimates at time $k$ are computed as

$$
\begin{aligned}
& \hat{\mu}_{k}=\sum_{i=1}^{N} \omega_{k}^{(i)} \hat{\mu}_{k}^{(i)} \\
& \widehat{\Sigma}_{k}=\sum_{i=1}^{N} \omega_{k}^{(i)}\left(\frac{\nu_{k}^{(i)}-d+1}{\nu_{k}^{(i)}-d-1} \Lambda_{k}^{(i)}+\left(\mu_{k}^{(i)}-\hat{\mu}_{k}\right)\left(\mu_{k}^{(i)}-\hat{\mu}_{k}\right)^{\mathrm{T}}\right)
\end{aligned}
$$

where the weights are inherited from the particles.

\section{EXPERIMENTAL RESULTS}

The presented method is evaluated on a number of real data sets collected with standard passenger cars under normal driving conditions. First a detailed analysis of one data set is presented in Section IV-A, followed by a statistical evaluation 
TABLE I

Pseudo CODE OF THE ALgORITHM

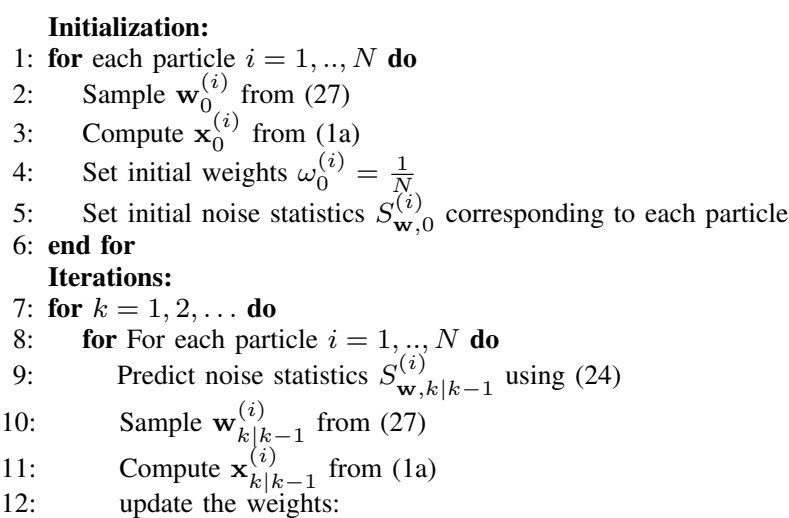

$$
\tilde{w}_{k}^{(i)}=w_{k-1}^{(i)} p\left(\mathbf{y}_{k} \mid \mathbf{x}_{k}^{(i)}\right)
$$

13: $\quad$ Update noise statistics $S_{\mathbf{w}, k}^{(i)}$ using (23)

14: end for

15: $\quad$ Normalize weights, $w_{k}^{(i)}=\frac{\tilde{w}_{k}^{(i)}}{\sum_{i=1}^{N} \tilde{w}_{k}^{(i)}}$.

16: Resample the particles.

17: Compute state estimate $\hat{\mathbf{x}}=\sum_{i=1}^{N} w_{k}^{(i)} \mathbf{x}_{k}^{(i)}$

18: Compute the parameter estimates using (30) 19: end for

of 22 data sets in Section IV-B (including two different vehicles and 12 different tire sets). In all cases both the detection of a tire deflation is shown as well as the robustness, i.e., that no false alarm is triggered when no deflation occurs over a significant timespan. The proposed marginalized particle filter (MPF) is compared with the extended Kalman filter's (EKF) estimates of the augmented state (10), which is denoted AUG$\mathrm{KF}$ in this section.

\section{A. Detailed Analysis of One Data Set}

In the experiments, the measurements were collected with a passenger car equipped with standard vehicle sensors, such as the ABS wheel speed sensors, and a GPS receiver, see Fig. 2. The sampling time of the wheel speed sensors is $0.1 \mathrm{~s}$ and the sampling time of the GPS receiver is 0.25 s, i.e., the prediction is performed more often than the measurement update. The standard deviation of the GPS measurements is set to $20 \mathrm{~m}$, however in reality it is probably much smaller.

In regions where the car moves at low velocities (less than $\gamma=11 \mathrm{~m} / \mathrm{s}$ ), the steering wheel angle measurement was utilized in order to avoid quantization problems of the wheel cogs in the third row of the motion model (7c), according to

$$
\psi_{k+1}= \begin{cases}\psi_{k}+T\left(\dot{\psi}_{k}^{\text {virt }}+\frac{\omega_{4} \delta_{4}}{l_{T}}-\frac{\omega_{3} \delta_{3}}{l_{T}}\right) & \text { if } \mathrm{v}>\gamma \\ \psi_{k}+T \delta_{F}\left(\mathrm{v}_{k}^{\mathrm{virt}}+\frac{\omega_{3} \delta_{3}}{2}+\frac{\omega_{4} \delta_{4}}{2}\right) / l_{b} & \text { if } \mathrm{v}<\gamma\end{cases}
$$

The filter is shut off completely at very low velocities (less than $7 \mathrm{~m} / \mathrm{s}$ ).

The GPS measurements of the $12 \mathrm{~km}$ test circuit is shown as a red solid line in Fig. 3. It is overlayed by the estimated trajectory, which is black-white dashed. The photo is a very accurate flight photo (obtained from the Swedish mapping,

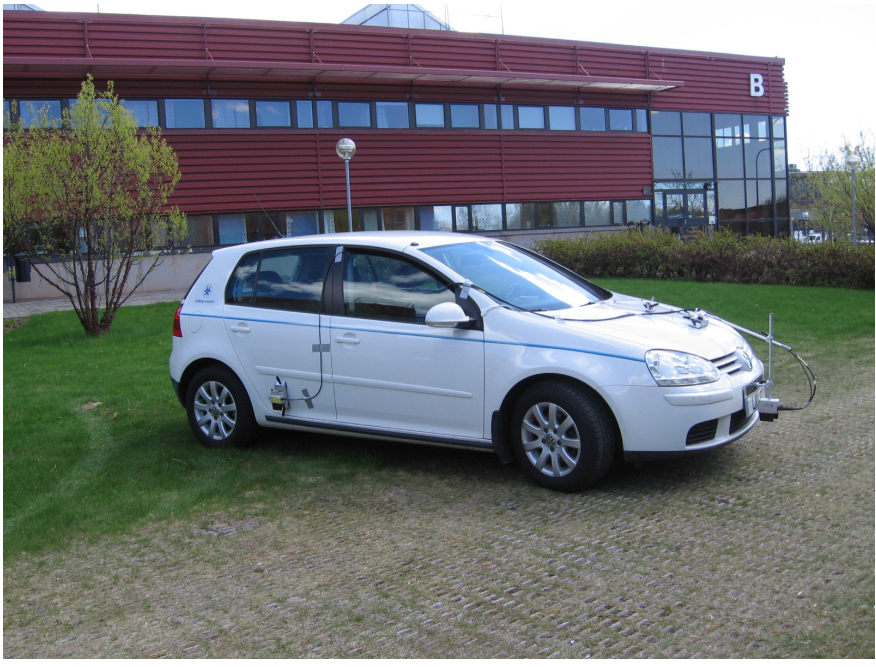

Fig. 2. The test vehicle of Linköping University is logging standard CAN data. The vehicle is in addition equipped with a GPS receiver.

cadastral and land registration authority), which can be used as ground truth to visualize the quality of the trajectory estimate. The circuit took about $18 \mathrm{~min}$ to drive and it starts and ends in urban area of Linköping, in the upper right corner in Fig. 3. The test vehicle is driving clockwise, first on a small rural road, and then on the left side of the figure entering a straight national highway, before driving back to urban area on the top of the figure. The test was performed two times, first with balanced tire pressure and thereafter with unbalanced tire pressure, where the pressure of the rear left tire was released to be approximately $50 \%$ of the right tire pressure.

For the first circuit the pressure of the rear wheel tires was adjusted to be equal 2.8 bar on both tires. The estimated mean parameter for the left and the right wheel are shown in the Fig. $4 \mathrm{a}$ and $4 \mathrm{~b}$, respectively. The MPF method, shown as a black solid line, performs well in estimating the mean values $\mu_{3}$ and $\mu_{4}$, and it is clearly visible that the mean values are similar and close to zero. The augmented model with EKF (AUG-KF) performs less well; it takes longer time to reach its end-value and is then moving around zero.

For the second circuit the pressure of the rear left tire was released to 1.5 bar. Comparing Fig. 4c with Fig. 4d it is visible that the pressure reduction leads to a smaller $\mu_{3}$ than $\mu_{4}$ value. Note that both methods reach the same end-value, approximately $1.5 \mathrm{~mm}$, but the proposed MPF is much faster and more stable than the AUG-KF method.

\section{B. Statistical Analysis Based on Multiple Data Sets}

When evaluating pressure loss due to puncture or diffusion this is usually carried out using step deflations. Hence, we have a controlled way of evaluating performance for a calibration phase where the nominal wheel radius can be estimated followed by a detection phase. In order to evaluate the performance there are two standardized test procedures, FMVSS138 NHTSA for the US market and ECE R-64 for the European market. In the experiments presented in this paper we use 22 ECE R-64 test cases, using two different vehicles 


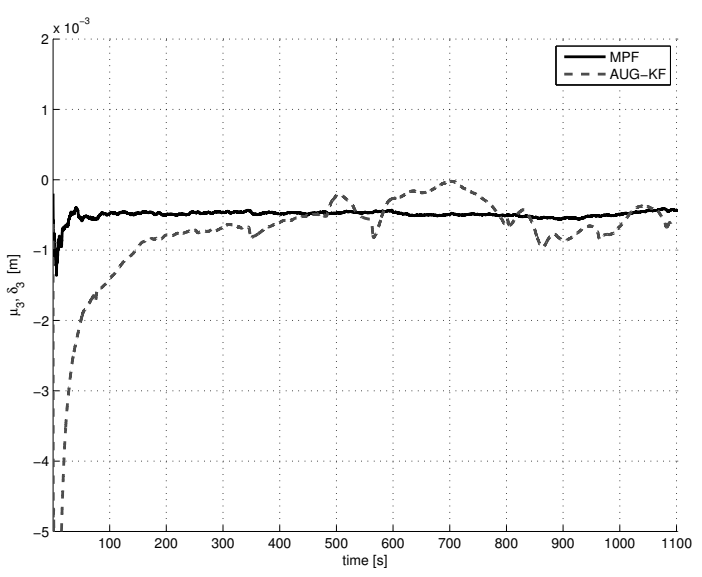

(a) Left Wheel

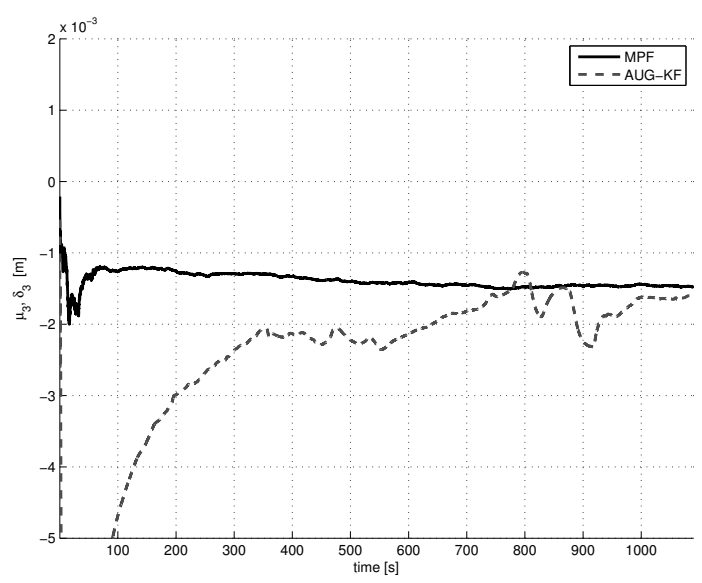

(c) Left Wheel, deflated

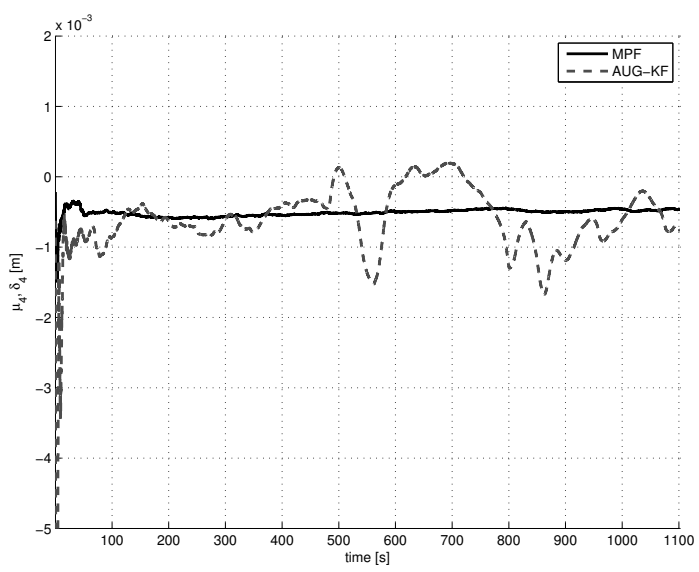

(b) Right Wheel

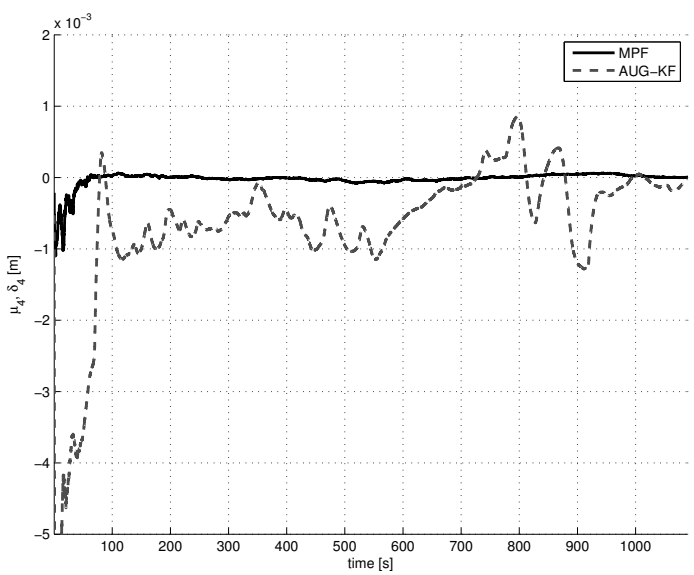

(d) Right Wheel

Fig. 4. Tire radius error of the left and right rear wheels. Fig. (a) and (b) show the situation with balanced wheel pressure and as expected the radius error is similar and close to zero. Fig. (c) and (d) show the situation with unbalanced wheel pressure, and the radius error of the left wheel in Fig. (c) is larger than the error of the right wheel in (d). The black solid line is the MPF estimate and the gray dashed line is the AUG-KF estimate.

from the same platform and 12 different tire sets. In this paper we focus on one-wheel puncture deflations on the non-driven wheels. The presented filters and approach can be applied to multiple wheel deflations and four-wheel driven vehicles if the model is extended and an appropriate slip compensation is included.

The standardized ECE R-64 test procedure constitutes of a calibration phase, in which the car is driving in the interval $40-120 \mathrm{~km} / \mathrm{h}$ for minimum $20 \mathrm{~min}$, followed by a step deflation of $20 \%$ from the pressure measured at deflation time. During the calibration and deflation, driving time is only considered when driving within the speed interval, excluding braking or extreme dynamic driving.

The data collected consists of GPS longitudinal and lateral information with a sampling rate of $0.5 \mathrm{~Hz}$. In addition wheel speed and yaw rate measurements as well as brake information are available in $10 \mathrm{~Hz}$. Note that we have focused on the more difficult problem in the current publication, where only position measurements are available from the GPS. If GPS Doppler velocity is measured the performance can be enhanced, but this signal is not always available on the CAN bus.

The ECE R-64 deflation scenario data is used to evaluate the performance of the radius estimator and the detector. In order to ensure sufficient robustness of the system, analysis is also performed on non-deflated robustness data. In this paper this is carried out re-using the same data and splitting the non-deflated data file in two. Hence the first part is used for calibration and the second part for detection. The goal is to have fast detection when a deflation is present and minimizing the risk of false alarms when robustness data is used.

The detector used in this paper is a standard CUSUM detector, [26], with some extensions, see Table II, where estimated radius error $\mu_{i, k}$ for each wheel $i$ and time $k$ is compared against its calibrated value. The implemented CUSUM uses a drift parameter $\nu$ and an adaptive threshold $\rho_{t h}$. To make it more robust against outliers in the input signal $\left(\mu_{i, k}\right)$, is formed by low pass filtering the difference in radius compared to calibration and apply a simple rate limiter. In the evaluation the following nominal parameters are used: $\nu=0.4 \cdot 10^{-3}$ and $\rho_{t h}=9 \cdot 10^{-3}$.

1) MPF - Performance evaluation: An example of the radius estimation and radius uncertainty estimation is depicted in Fig. 5 for a step deflation. As seen, the deflation is easily detected. In order to analyze the performance, the detection 


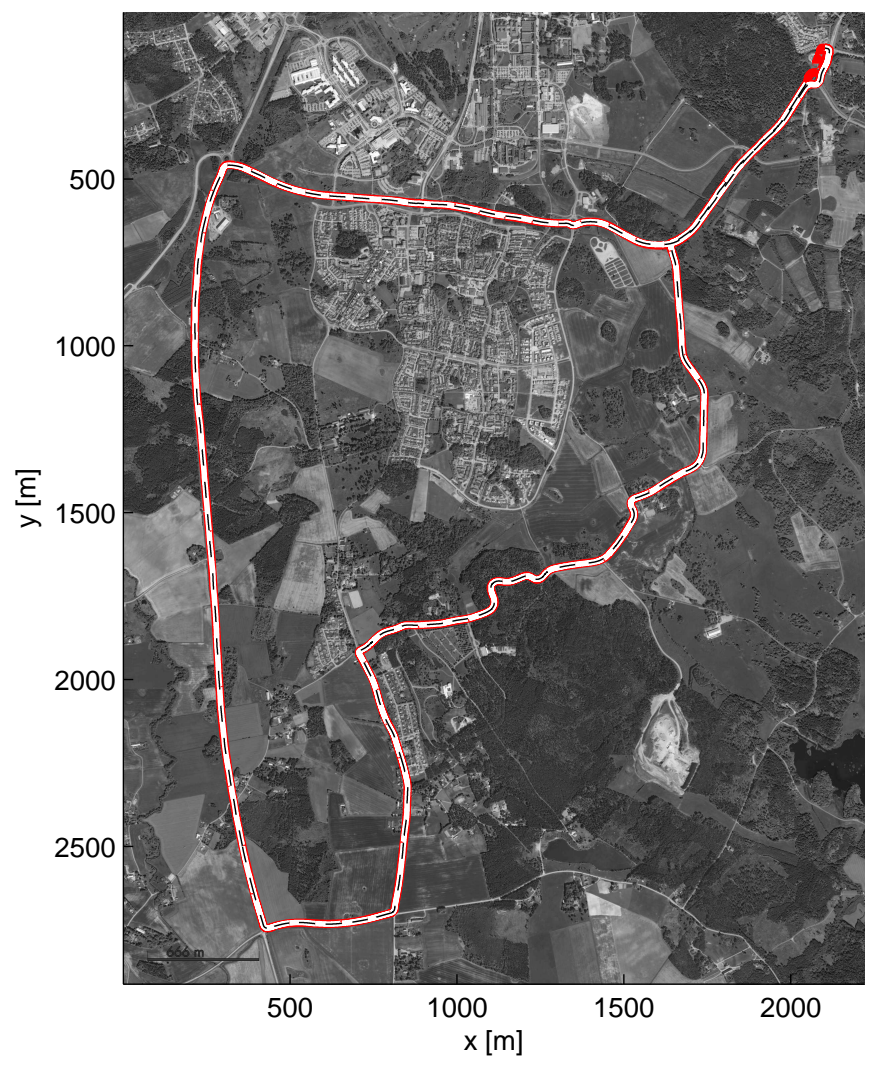

Fig. 3. The red line is GPS position measurements and the black-white dashed line is the estimated driven trajectory. The experiment starts and ends at a roundabout in the upper right corner. The resolution of this photo is one square meter per pixel. (C)Lantmäteriet Medgivande I2011/1405, reprinted with permission)

TABLE II

CUSUM PSEUDO CODE FOR TIRE DEFLATION ALARM.

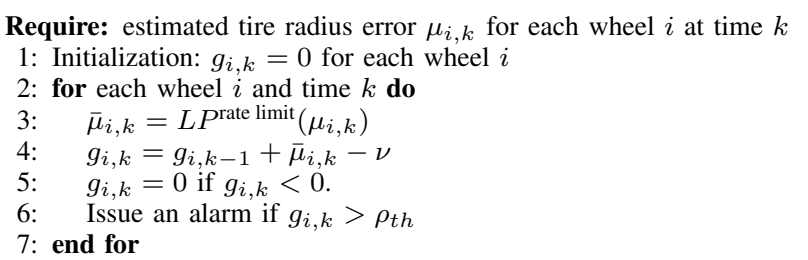

times are analyzed using a performance evaluation of 22 one-wheel deflations. The MPF was able to detect 21 of the deflations using a CUSUM detector, considering $\mu_{i, k}$, i.e., the calibrated radius versus the current estimated radius, as a difference signal for each wheel. The test case with a missed detection was very close to trigger an alarm. In Fig. 6 the detection time histogram for the MPF is depicted and as seen most of the detections are below 10 minutes which complies with the requirements for a one-wheel deflation, according to ECE R-64. Note, that if the aim only was to detect a one-wheel detection, the fastest method would be to compare the wheel speeds between all wheels of the vehicle, now every wheel is treated separately. Using the presented method it is also possible to detect diffusion (a slow reduction of air pressure) up to four wheels, which allows a detection time of 60 minutes according to the specification. A histogram plot of deflation

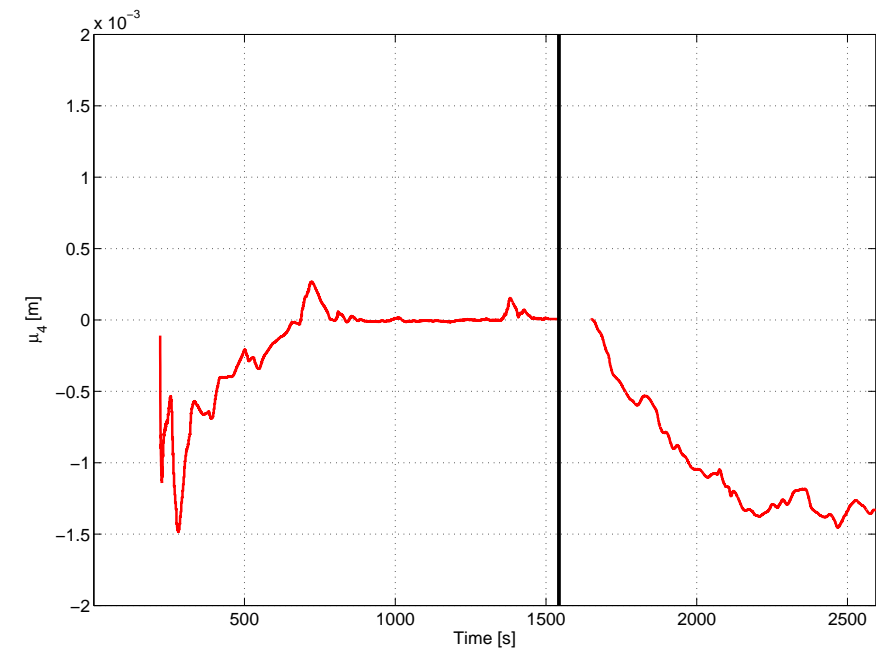

Fig. 5. An example of radius and radius uncertainty estimation using the MPF. First the calibration part is depicted followed by the step deflation part. As seen the change in radius is essential.

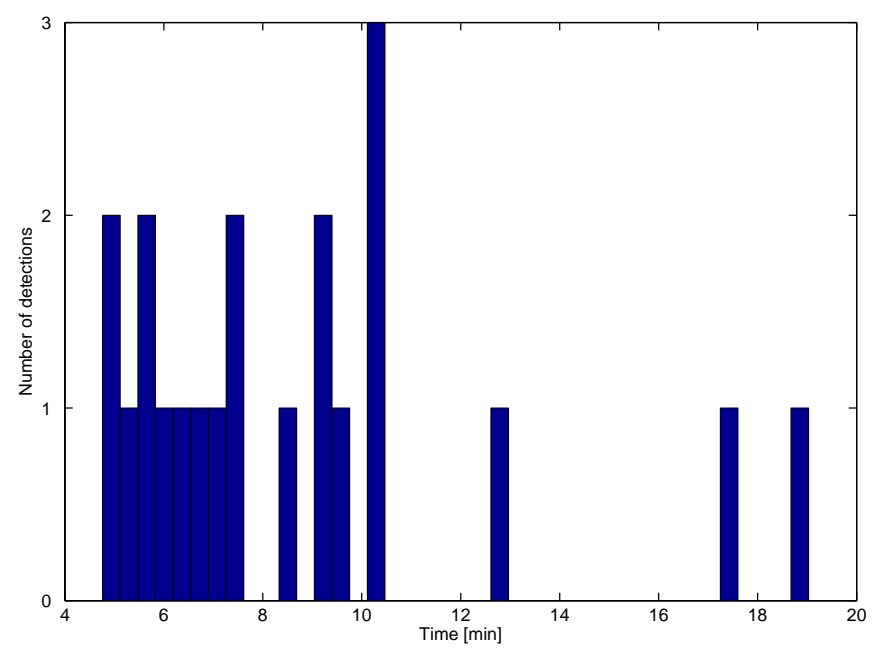

Fig. 6. The figure shows the detection histogram for the MPF. One missed detection not shown.

estimates $\Delta$ is depicted in Fig. 8. The detection and robustness statistics are summarized in Table III.

2) AUG-KF - Performance evaluation: The AUG-KF performance is significantly worse than the MPF, only detecting 16 of the 22 deflations correctly. The detection time histogram is presented for the AUG-KF in Fig. 7 and the detection and robustness statistics are summarized in Table III. The better performance of the MPF can be explained by the particle filter's ability to restrict parameter estimates to only allow decrease of tire radius after calibration and that it can handle nonlinearities optimally. Furthermore, the MPF method distinguishes better between the parameters (wheel radii) and the position state variables. The disable criteria, the motion model, the noise variables and the CUSUM parameters are equal, or in the cases where the model structure not comprises the same parameter set as similar as possible, for the two compared methods. 


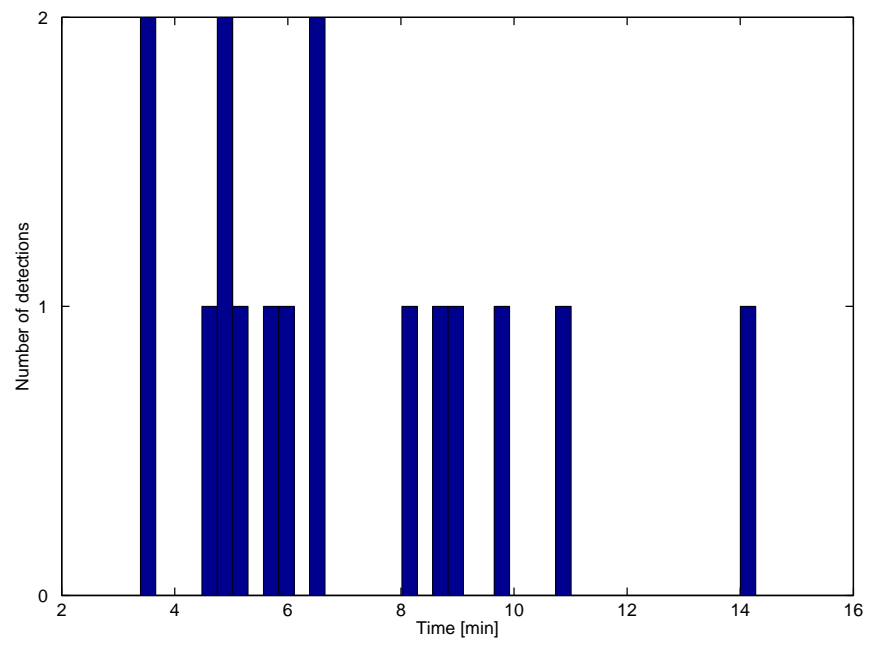

Fig. 7. The figure shows the detection histogram for the AUG-KF. Six missed detections not shown.

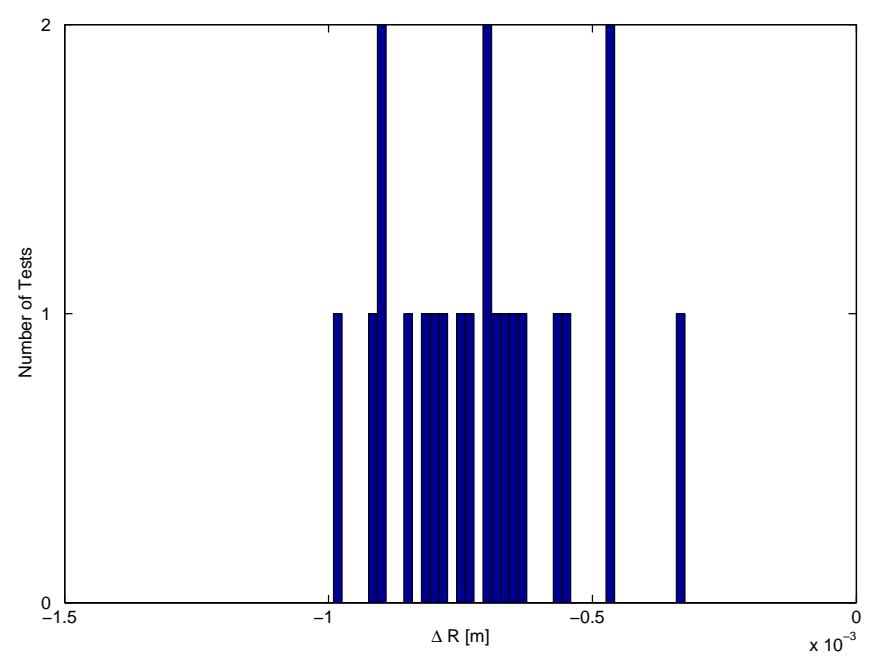

Fig. 8. The figure shows a histogram of estimated wheel radius differences $\mu_{i, k}$ due to tire deflation. Tires from different manufacturers were used, which can explain the spread.

\section{CONCLUSION}

In this study, we address the problem of joint estimation of unknown tire radii and the trajectory of a four-wheeled vehicle based on GPS and wheel angular velocity measurements. The problem is defined in a Bayesian framework and an efficient method that utilizes marginalized particle filters is proposed in order to accomplish the difficult task of joint parameter and state estimation. The algorithm is tested on real data experiments performed in accordance with the regulation 64 of the United Nations Economic Commission for Europe (ECE R64). The results show that it is possible to estimate the separate

TABLE III

DETECTION AND FALSE ALARM STATISTICS FOR MPF AND AUG-KF USING 22 TESTS.

\begin{tabular}{|l|l|l|}
\hline & Detection [\%] & False Alarm [\%] \\
\hline PF & 95.5 & 0.0 \\
\hline EKF & 72.7 & 0.0 \\
\hline
\end{tabular}

tire radius within sub-millimeter accuracy .

\section{ACKNOWLEDGMENTS}

The authors would like to thank Kristoffer Lundahl, at the vehicular systems division at Linköpings University, for assisting with the data collection and Nira Dynamics for providing data used for estimation and statistical analysis.

\section{REFERENCES}

[1] S. Velupillai and L. Guvenc, "Tire pressure monitoring," IEEE Control Systems Magazine, vol. 27, no. 6, pp. 22-25, Dec. 2007.

[2] N. Persson, S. Ahlqvist, U. Forssell, and F. Gustafsson, "Low tire pressure warning system using sensor fusion," in Proceedings of the Automotive and Transportation Technology Congress, ser. SAE paper 2001-01-3337, Barcelona, Spain, Oct. 2001.

[3] S. L. Miller, B. Youngberg, A. Millie, P. Schweizer, and J. C. Gerdes, "Calculating longitudinal wheel slip and tire parameters using GPS velocity," in IEEE American Control Conference, vol. 3, Jun. 2001, pp. 1800-1805.

[4] C. R. Carlson and J. C. Gerdes, "Consistent nonlinear estimation of longitudinal tire stiffness and effective radius," IEEE Transactions on Control Systems Technology, vol. 13, no. 6, pp. 1010-1020, Nov. 2005.

[5] N. M'sirdi, A. Rabhi, L. Fridman, J. Davila, and Y. Delanne, "Second order sliding mode observer for estimation of velocities, wheel sleep, radius and stiffness," in IEEE American Control Conference, Jun. 2006, pp. 3316-3321.

[6] H. Shraim, A. Rabhi, M. Ouladsine, N. M'Sirdi, and L. Fridman, "Estimation and analysis of the tire pressure effects on the comportment of the vehicle center of gravity," in International Workshop on Variable Structure Systems, Jun. 2006, pp. 268-273.

[7] A. Censi, L. Marchionni, and G. Oriolo, "Simultaneous maximumlikelihood calibration of odometry and sensor parameters," in Proceedings of the IEEE International Conference on Robotics and Automation, Pasadena, Canada, May 2008, pp. 2098-2103.

[8] S. Patwardhan, H.-S. Tan, and M. Tomizuka, "Experimental results of a tire-burst controller for AHS," Control Engineering Practice, vol. 5, no. 11, pp. 1615-1622, 1997.

[9] V. E. Ersanilli, P. J. Reeve, K. J. Burnham, and P. J. King, "A continuoustime model-based tyre fault detection algorithm utilising a Kalman state estimator approach," in Proceedings of the 7th Workshop on Advanced Control and Diagnosis, Zielona Góra, Poland, Nov. 2009.

[10] N. J. Gordon, D. J. Salmond, and A. Smith, "A novel approach to nonlinear/non-Gaussian Bayesian state estimation," in IEE Proceedings on Radar and Signal Processing, vol. 140, 1993, pp. 107-113.

[11] B. Ristic, S. Arulampalam, and N. Gordon, Beyond the Kalman Filter: Particle Filters for Tracking Applications. Artech House, 2004.

[12] A. Doucet, N. de Freitas, and N. Gordon, Eds., Sequential Monte Carlo Methods in Practice. Springer Verlag, 2001.

[13] B. Anderson and J. B. Moore, Optimal Filtering. Englewood Cliffs, NJ: Prentice Hall, 1979.

[14] A. H. Jazwinski, Stochastic Processes and Filtering Theory, ser. Mathematics in Science and Engineering. Academic Press, 1970, vol. 64.

[15] J. Liu and M. West, "Combined parameter and state estimation in simulation-based filtering," in Sequential Monte Carlo Methods in Practice, A. Doucet, N. D. Freitas, and N. Gordon, Eds. Springer, 2001.

[16] S. Saha, E. Özkan, F. Gustafsson, and V. Smidl, "Marginalized particle filters for Bayesian estimation of Gaussian noise," in Proceedings of the International Conference on Information Fusion, Edinburgh, Scotland, Jul. 2010.

[17] E. Özkan, C. Lundquist, and F. Gustafsson, "A Bayesian approach to jointly estimate tire radii and vehicle trajectory," in Proceedings of the IEEE Conference on Intelligent Transportation Systems, Washington DC, USA, Oct. 2011.

[18] U. Kiencke and L. Nielsen, Automotive Control Systems, 2nd ed. Berlin, Heidelberg, Germany: Springer, 2005.

[19] J. Y. Wong, Theory Of Ground Vehicles, 3rd ed. New York, USA: John Wiley \& Sons, 2001.

[20] F. Gustafsson, S. Ahlqvist, U. Forssell, and N. Persson, "Sensor fusion for accurate computation of yaw rate and absolute velocity," in Proceedings of the SAE World Congress, ser. SAE paper 2001-01-1064, Detroit, MI, USA, Apr. 2001. 
[21] F. Gustafsson, Statistical Sensor Fusion. Lund, Sweden: Studentlitteratur, 2010

[22] W. Rugh, Linear System Theory. Upper Saddle Rivers, NJ: Prentice Hall, 1996.

[23] A. Doucet, S. J. Godsill, and C. Andrieu, "On sequential Monte Carlo sampling methods for Bayesian filtering," Statistics and Computing, vol. 10, no. 3, pp. 197-208, 2000.

[24] V. Peterka, "Bayesian system identification," Automatica, vol. 17, no. 1, pp. 41-53, Jan. 1981

[25] M. Kárný, Optimized Bayesian Dynamic Advising: Theory and Algorithms. London: Springer, 2006.

[26] F. Gustafsson, Adaptive Filtering and Change Detection. Chichester, West Sussex, England: John Wiley \& Sons, 2000.

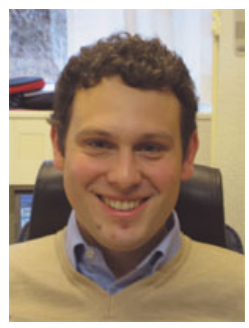

Christian Lundquist received the M.Sc. degree in Automation and Mechatronics Engineering from Chalmers University of Technology, Gothenburg, Sweden, in 2003. He received the Ph.D. degree in 2011, at the Department of Electrical Engineering at Linköping University, Linköping, Sweden, where he now works as a postdoctoral fellow.

Between 2004 and 2007, he worked on active steering systems at ZF Lenksysteme $\mathrm{GmbH}$, Germany. His research interests include sensor fusion and target tracking for automotive applications.

$\mathrm{He}$ is CEO and co-founder of the company SenionLab, developing navigation and positioning solutions for indoor usage.

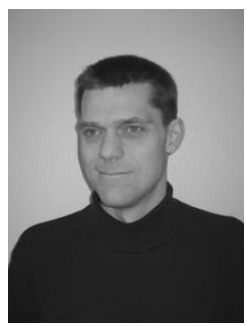

Rickard Karlsson received the M.Sc. degree in applied physics and electrical engineering in 1996, and a Ph.D. in automatic control in 2005, both from Linköping university, Linköping, Sweden. He worked at Saab Bofors Dynamics in Linköping between 1997 and 2002, on sensor fusion and target tracking, between 1999 and 2002 was partly employed at the Automatic Control group, and from 2002 full time. During 2005-2007 he was Assistant Professor at the Automatic Control group in Linköping. He worked for NIRA Dynamics 20072010 and from 2012, developing sensor fusion systems for automotive applications, and during 2010-2012 he worked for the Swedish Defence Research Agency (FOI) as competence leader in sensor fusion. He received an Associate Professor in Automatic control in 2010.

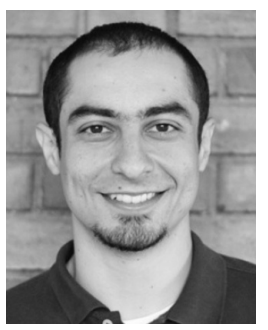

Emre Özkan received the B.S. and Ph.D. degrees in electrical engineering from Middle East Technical University, Ankara, Turkey, in 2002 and 2009 respectively. From 2002 to 2008 , he was with the Department of Electrical and Electronics Engineering, Middle East Technical University, as a Teaching and Research Assistant. From 2009 to 2011 he was a Postdoctoral Associate with the Division of Automatic Control, Department of Electrical Engineering, Linköping University, Linköping, Sweden. Currently he is an Assistant Professor in the same department. His research interests include statistical signal processing, estimation theory, sensor fusion, and target tracking.

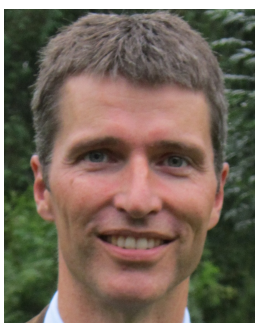

Fredrik Gustafsson (S'91-M'93-SM'05) received the M.Sc. degree in electrical engineering and the Ph.D. degree in automatic control, both from Linköping University, Linköping, Sweden, in 1988 and 1992, respectively.

From 1992 to 1999 , he held various positions in automatic control, and from 1999 to 2005 he had a professorship in communication systems at Linköping University, where he has been a Professor in sensor informatics at the Department of Electrical Engineering since 2005. His research interests are in stochastic signal processing, adaptive filtering, and change detection, with applications to communication, vehicular, airborne, and audio systems. His work in the sensor fusion area involves design and implementation of nonlinear filtering algorithms for localization, navigation, and tracking of all kind of platforms, including cars, aircraft, spacecraft, UAV's, surface and underwater vessels, cell phones, and film cameras for augmented reality. He is a cofounder of the companies NIRA Dynamics and Softube, developing signal processing software solutions for automotive and music industry, respectively.

Dr. Gustafsson was an Associate Editor for the IEEE TRANSACTIONS OF SigNAL PROCESSING from 2000 to 2006 and is currently Associate Editor for the EURASIP Journal on Applied Signal Processing and International Journal of Navigation and Observation. In 2004, he was awarded the Arnberg prize by the Royal Swedish Academy of Science (KVA), and in 2007 he was elected member of the Royal Academy of Engineering Sciences (IVA). 